Revue d'histoire de l'Amérique française

ZBS REVUE D.HISTOIRE DE L'AMÉRIQUE FRANÇAISE

\title{
L'évolution économique du Canada et du Québec depuis la Confédération
}

\section{François-Albert Angers}

Volume 21, numéro 3a, 1967

Cent ans d'histoire 1867-1967

URI : https://id.erudit.org/iderudit/302715ar

DOI : https://doi.org/10.7202/302715ar

Aller au sommaire du numéro

Éditeur(s)

Institut d'histoire de l'Amérique française

ISSN

0035-2357 (imprimé)

1492-1383 (numérique)

Découvrir la revue

Citer cet article

Angers, F.-A. (1967). L'évolution économique du Canada et du Québec depuis la Confédération. Revue d'histoire de l'Amérique française, 21(3a), 635-655.

https://doi.org/10.7202/302715ar d'utilisation que vous pouvez consulter en ligne.

https://apropos.erudit.org/fr/usagers/politique-dutilisation/ 
François-Albert Angers

L'ÉVOLU'TION ÉCONOMIQUE

DU CANADA

ET DU QUEBEC

DEPUIS LA

CONFÉDÉRATION 


\section{François-Albert Angers}

Economiste et professeur.

Né à Québec, le 21 mai 1909. Etudes au Collège des Frères du Sacré-Cœur, Montmagny, à l'Ecole des Hautes Etudes commerciales de Montréal, à l'Ecole libre des Sciences Politiques (Paris).

Professeur et directeur de l'Institut d'Economie appliquée à l'Ecole des Hautes Etudes commerciales. Canada.

Membre de la Commission française des Semaines sociales du

Aviseur économique de la Chambre de Commerce du District de Montréal.

Président de la Ligue d'Action nationale et directeur de sa revue jusqu'en ces derniers temps.

Membre de la Commission générale des Etats généraux du Canada français.

Administrateur de Les Placements collectifs Inc., La Solidarité, La Société nationale de Fiducie.

Oeuvres :

Initiation à l'économie politique, Fides, $4 \mathrm{e}$ édition

Essai sur la centralisation, Beauchemin et Ecole des Hautes Etudes commerciales

La sécurité sociale et les problèmes constitutionnels, 2 vol. Annexe au Rapport de la Commission Royale d'enquête du Québec sur les Problèmes constitutionnels

Le problème fiscal et les relations fédérales-provinciales, 1 v. Annexe au Rapport de la Commission, comme ci-dessus

Participation aux ouvrages collectifs de la Collection des "Etudes sur notre Milieu" de l'Ecole des Hautes Etudes commerciales

Diverses brochures ou études sur des problèmes économiques ou nationaux.

Nombreux articles à un nombre très varié de revues canadiennes et européennes, et tout particulièrement collaboration régulière et fréquente depuis 1938, sur les problèmes économiques à L'Actualité Economique, et sur les questions politiques, sociales et nationales à $L^{\prime}$ Action nationale. 


\section{L'ÉVOLUTION ÉCONOMIQUE DU CANADA ET DU QUEEBEC DEPUIS LA CONFÉDERRATION}

Bien des historiens l'ont dit, ce sont des motifs économiques qui auraient le plus convaincu les colonies britanniques de l'Amérique du Nord et de s'unir en fédération en 1867 et de se voir confier l'administration de ce qui est aujourd'hui l'immense territoire canadien. $\AA$ Londres, peut-être pensait-on bien davantage en termes de défense militaire, optique qui allait engendrer une lourde hypothèque financière et imposer une contrainte de développement économique de considérable portée dans l'évolution de l'économie canadienne. Mais la thèse de la "prospérité des provinces" mieux assurée par l'immensité des territoires unis était non moins dans les idées d'économie libérale de l'époque. Quand on pense aux quatre provinces qui ont constitué le noyau initial de cette association - l'Ontario, le Québec, le Nouveau-Brunswick et la Nouvelle-Écosse - il y aurait matière à épiloguer avec quelque ironie sur le danger d'une foi trop assurée dans les théories générales de l'économie, dont les vérités à portée universelle (?) sont souvent source de désappointement pour ceux qui en tirent conclusion pour les cas particuliers. Car de ces quatre provinces, il n'en est qu'une dont on puisse être sûr qu'elle a vraiment profité de la dimension du territoire canadien. Il est certain pour deux d'entre elles et probable pour la troisième, qu'elles ont beaucoup plus contribué au progrès de l'Ontario et des territoires alors non organisés qu'elles n'en ont tiré profit.

Mais il y a eu en tout cas un développement économique important sous le régime confédératif, dont il deviendrait fort hypothétique de vouloir démêler ce qui revient au régime politique de l'union des provinces selon les données de la théorie 
libérale des grands espaces, et ce qui se serait de toute façon produit quand même du seul fait du mouvement général du siècle. Même l'Ouest d'ailleurs montre qu'il doute des avantages que lui aurait valu le régime politique comme tel. Les facteurs politiques en jeu portent à croire que, sans la Confédération, ces provinces auraient été perdues pour le Commonwealth et auraient été jointes aux Etats-Unis. Or si l'Ouest s'est développé plus à l'intérieur de la Confédération qu'il aurait normalement dû le faire sans la construction de chemins de fer, à buts militaires, il n'est pas sûr qu'il n'aurait pas davantage profité de l'annexion aux Etats-Unis. Dans l'Ouest, on ne se plaint pas moins, ou à peine moins, que dans les Maritimes d'être retardé dans le développement par une politique qui avantage l'Est, du fait des barrières tarifaires avec les États-Unis. L'Ontario seul paraît avoir sûrement profité dans l'ordre économique de l'œuvre politique des Pères de la. Confédération.

Comme le Canada n'existerait pas sans la constitution de 1867, il faut bien concéder qu'il doit à cette constitution l'état de son développement économique comme ensemble identifié, même s'il n'est pas sûr que cet avantage ne soit réel que pour une seule de ses parties, ou même que la somme de la prospérité des parties selon le destin qui leur aurait été propre autrement ne serait pas plus grande. Il faudrait une analyse serrée pour mesurer la possibilité, pourtant réelle en l'occurrence, de cette dernière éventualité; car la dimension en elle-même comporte une sorte de mirage qui nous séduit au départ et nous rend invraisemblable l'idée que du plus petit puisse contenir plus que $\mathrm{du}$ plus grand. Pour ébranler les convictions trop assurées, rappelons la thèse d'André Siegfried sur la mauvaise construction géographique et économique que constitue la grande dimension canadienne, faite de vastes régions juxtaposées en est-ouest par une volonté politique qui a voulu les tenir reliées par une ligne de chemin de fer, alors que la vocation géographico-économique de chacune paraît nettement orientée plutôt selon l'axe nord-sud. 
Quoi qu'il en soit des théories et des thèses c'est de 1867 à 1967 que l'ensemble canadien s'est élevé du rang de colonie à celui de nation, au sens politico-juridique du terme; et du rang de pays sous-développé ou non-développé à celui de pays industriel jouissant de l'un des plus hauts standards de vie au monde. En 1867, la valeur de la production minérale du Canada était d'environ 20 fois inférieure à celle du produit des grandes cultures; alors que la production manufacturière - et d'ailleurs à un niveau artisanal de technique - n'en était que 2 fois plus grande. Aujourd'hui, la valeur de la production minérale dépasse la production des grandes cultures par environ $11 / 2$ fois en dépit de l'addition au Canada de 1867 des vastes provinces agricoles des Prairies; et la production manufacturière y est plus de $121 / 2$ fois supérieure. C'est tout un pays qui s'est bâti pendant cette période, avec la construction de ses chemins de fer, de ses canaux, de ses routes, de ses aéroports; la mise en valeur de tous les types de ressources, parmi lesquelles la nouvelle ressource moderne de l'énergie hydro-électrique a brillé avec une particulière intensité; l'établissement de tous les réseaux commerciaux et financiers caractéristiques d'un grand pays.

Dans l'absolu des données brutes, le Québec a connu un développement similaire. La précision des statistiques disponibles ne permet pas d'établir la comparaison directe avec les chiffres précédents pour le Canada. Mais il est bien évident que les écarts seraient plus considérables quant au Québec seul. Qu'il suffise de constater qu'en 1961, 9\% seulement de la maind'œuvre masculine y était engagée encore dans l'agriculture, contre $13 \%$ pour l'ensemble du Canada. Il est symptomatique toutefois qu'en dépit de sa richesse en ressources et de sa situation favorable à l'entrée du pays, le Québec, qui est la plus ancienne province, ne fasse pas le poids (environ $27 \%$ contre $29 \%$ ) dans sa part de la production manufacturière de l'ensemble du pays. Plus symptomatique encore est le fait que la partie vraiment développée du Québec, Montréal, avec $111 / 2 \%$ de la population du pays et $15 \%$ de la production industrielle, soit le port d'entrée maritime du reste du Canada, jusqu'à récemment stade obligatoire par la barrière au transport des rapides Lachine, 
maintenant partiellement surmontée par la canalisation du SaintLaurent. Située à l'extrême sud-ouest du Québec, cette ville annonce la prospérité de l'hinterland, laissant tout le reste du Québec avec $171 / 2 \%$ de la population du pays, mais seulement $12 \%$ de la production industrielle.

Enregistrons pour le moment ces constatations relatives à l'ensemble du Québec et considérons l'évolution économique du Canada lui-même. Il ne s'est pas révélé capable encore, au cours de ces cent ans de dégagement de son indépendance politique, de se donner un développement économique vraiment autonome; autonome ici n'ayant en aucune façon le sens d'économie fermée, autarcique, mais d'un développement de maturité, qui ne serait conditionné que par la valorisation maximum des ressources selon le seul intérêt national, où l'échange international est envisagé librement comme un appoint supplémentaire négocié sur une base d'égalité et de réciprocité dans les avantages mutuels.

C'est sur une base coloniale que s'est amorcé le développement économique du Canada, c'est-à-dire l'intervention de l'investissement métropolitain selon les intérêts de la métropole. Mais au tournant du siècle, un troisième partenaire est intervenu dans le jeu des forces politiques et économiques: le voisin américain. Il a profité à la fois des hésitations d'une politique canadienne mal définie en raison de l'allégeance britannique, de ses jeux diplomatiques avec la métropole et des avantages qu'il en a tirés aux dépens des points de vue canadiens, ainsi que du désir des Canadiens de pouvoir participer au plus vite à la prospérité générale du continent nord-américain. Ainsi le Canada s'est-il en quelque sorte donné à une seconde métropole, ligne du moindre effort pour s'assurer un haut standard de vie sans avoir à affronter les risques ordinaires de l'investissement national et de la concurrence sur les marchés internationaux.

Ainsi le petit Canada, en termes de population, est-il devenu le cinquième pays exportateur du monde, mais pour alimenter principalement le développement économique d'une seule puissance, les États-Unis, qui en absorbe les 70\%. Les liaisons sur lesquelles repose ce résultat revêtent toute leur signification dans 
le fait qu'à l'heure actuelle, $59 \%$ des investissements industriels, $69 \%$ des investissements pétroliers et $59 \%$ des investissements miniers sont sous contrôle étranger, avec $45 \%, 69 \%$ et $52 \%$ respectivement sous contrôle américain. Et ces pourcentages, pourtant impressionnants, minimisent quand même la véritable portée de la domination étrangère sur l'économie canadienne. Pour le montrer, il faudrait entrer dans les détails qui illustreraient comment l'investissement étranger occupe le cœur de l'économie, les pôles du développement dont dépend l'activité du résidu d'investissement canadien, qui se branche sur leurs effets d'entraînement. Et avec le passage des années, ce phénomène ne s'est pas atténué, marquant la marche vers une maturité; il s'est au contraire accentué.

Il n'est pas impliqué que ce processus interdit tout accès à la phase de maturité; mais comme l'intérêt national ne cesse d'avoir sa signification dans les relations économiques, envers et contre toutes les théories générales qui en font abstraction, cette maturité ne surviendra ainsi qu'à travers un plus long processus au bout duquel le problème se résoudra par l'intégration totale de l'économie canadienne dans l'économie américaine. En attendant, ce n'est pas uniquement par hasard et par sa "vocation" propre, dans le sens de la théorie classique du commerce international, que le Canada ne jouit que d'un développement industriel de type secondaire ou accessoire à sa satellisation; qu'il reste un pays fournisseur de produits agricoles et de matières premières brutes ou sommairement ouvrées. C'est bel et bien parce qu'il n'est pas sorti du stade de colonie d'exploitation, ici sublimée, qui "glane" pour son développement industriel les résidus d'activité qu'engendrent les besoins et les exigences d'une action principale tout entière ordonnée selon les intérêts d'un autre espace organisé autour d'une autre capitale politique qu'Ottawa, autour de Washington.

Mais revenons au Québec, dont nous avons vu qu'il ne semble pas avoir retiré de ce mouvement la part à laquelle on aurait pu 
s'attendre. Cela doit être relié à un grand fait de l'histoire économique canadienne qui a tout conditionné, qui explique à peu près tout, et dont on ne tire généralement que les conséquences banales: celui de la construction des chemins de fer au Canada.

En lui-même, ce fait est assez banal. Partout où il y eut à effectuer la mise en valeur d'un territoire neuf à l'époque moderne, la construction des chemins de fer a joué un rôle majeur, parce que c'était le mode de transport miracle de l'époque, et que l'organisation des moyens de transport, toujours et de tout temps, a été la condition du développement économique. Mais au Canada le fait s'est développé selon un mode inusité, entraînant des conséquences spéciales sur l'orientation du développement économique des derniers cent ans. Sauf en Russie soviétique, où l'on a fait quelque chose du genre du côté de la Sibérie - mais alors d'une façon consciente et bien située dans un plan -, il ne semble pas qu'aucun autre pays au monde ne se soit ainsi donné des milliers de milles de chemin de fer, tout d'un coup, à travers tout le territoire vide d'un continent. Aussi bien, à l'époque de pensée libérale dans laquelle a pris naissance la Confédération, cette politique inusitée ne fut-elle pas dictée par des impératifs économiques, mais par les impératifs de défense militaire qui faisaient désirer cette union des provinces aux dirigeants londoniens.

L'observation de la mise en valeur de pays comme les ÉtatsUnis, le Brésil, l'Argentine, l'Australie, dont l'histoire peut se comparer à celle du Canada, révèle que les populations se sont concentrées d'abord au point d'arrivée, qu'elles ont tendu à développer au maximum les régions limitrophes afin d'assurer au coût minimum le rendement maximum des premiers investissements avant de s'étendre plus loin dans l'espace libre. Ainsi le mouvement démographique et la mise en valeur du territoire font-ils progressivement tache d'huile. De même on n'ouvre qu'au fur et à mesure de nouvelles régions, et on n'y construit les chemins de fer, que déjà précédés par des pionniers débordant de la vague de population installée sur la partie déjà mise en valeur. 
Une carte historique par points de la distribution de la population fait ressortir au Canada une tout autre histoire: une pénétration rapide de tout le territoire canadien, "a mari usque ad mare", en un long ruban de population dispersée de part et d'autre d'une ligne qui n'est autre que la voie du chemin de fer transcontinental. N'épiloguons pas ici sur les charges invraisemblables que cet investissement énorme a imposées comme un boulet au pied au développement d'une économie proprement canadienne. Mais voyons la conséquence qui en découlait inévitablement, surtout dans un cadre de pensée libérale. Il restait maintenant à valoriser au rnaximum cet investissement, donc à établir le long de cette voie plutôt qu'ailleurs toute la population canadienne; non pas seulement pour permettre aux chemins de fer d'en tirer des revenus -- ce qui n'est que le signe extérieur du problème fondamental - - mais surtout pour n'avoir pas à encourir de nouvelles dépenses d'investissement afin de développer d'autres parties du territoire avant que n'ait atteint son point de saturation celle qui se trouvait ainsi ouverte. Ce plan non planifié selon une vision économique du développement - et encore bien davantage parce que n'étant le résultat d'aucune planification, il devait ensuite s'intégrer dans un fonctionnement libéral de l'économie - condamnait formellement le Québec à être en quelque sorte abandonné pour une période indéterminée, dont le dessin général s'exprimerait ainsi: développer d'abord tout le reste du Canada à l'ouest de Montréal, puis retour au Québec ensuite seulement, quand le développement du reste du Canada aura permis la réalisation d'un état normal de rentabilité des investissements déjà engagés dans l'Ouest.

Ainsi se trouvait établi, sans qu'il ait été à proprement parler préconçu et pour être réalisé par la force des choses, un plan de développement du Canada qui comportait l'utilisation de Montréal comme port d'entrée, la vallée ontarienne des Grands Lacs comme pôle géographique de développement, avec projection de ses rayonnements vers l'Ouest dans son souci d'expansion. Le Québec n'allait plus entrer dlans ce plan, au lieu d'un développement intensif du type de celui de la Nouvelle-Angleterre qui aurait normalement été son lot, que dans la mesure où il était 
nécessaire d'y passer; et le long des lignes de passage pour aller vers le centre. La rive sud du Saint-Laurent a effectivement profité des promesses faites à Halifax de le relier au transcontinental; Sherbrooke s'est situé sur la ligne des relations avec les Etats-Unis; l'Abitibi et notre développement minier ne se sont trouvés que par hasard sur l'une de ces lignes, dont l'histoire politique semble montrer qu'elle n'a pas été située selon la meilleure direction d'ensemble, qui aurait été la direction préférable pour le Québec (Saint-Félicien, Chibougamau, rives de la baie James, etc., vers l'Ouest), en vue de favoriser le développement minier du Nord-Ontario.

Seul le développement de la région du lac Saint-Jean-Chicoutimi a échappé à la règle, à cause de l'audace des 21 de Charlevoix et de l'esprit d'entreprise de sir William Price. Ici nous avons vu un mode traditionnel de développement se produire par l'entêtement de pionniers qui forceront ensuite la venue du chemin de fer - et on ne leur a pas construit la meilleure ligne de chemin de fer au Canada! Et rien n'a ensuite été fait convenablement pour faire de cette région le pays actif qu'elle aurait été si elle s'était trouvée reliée plutôt à Chibougamau et au lac Albanel et si on y avait rattaché l'Abitibi, qui y aurait trouvé là son débouché le plus près de l'accès à la mer, au lieu d'en organiser les relations dans l'axe des intérêts miniers de Toronto.

Dans ce vaste Canada, Québec a donc été comme un pays abandonné à lui-même, sans aide et sans appui; laissé aux paysans qui s'y étaient établis comme en réserve, en attendant que la mise en valeur du reste du pays terminée, ou suffisamment avancée, on puisse songer à reconquérir cette paysannerie pour l'intégrer dans le grand tout canadien, qui alors se ferait plus agressif parce qu'il aurait besoin de ses ressources.

Ne parlons pas de plan préconçu, machiavélique. Les AngloSaxons n'ont pas besoin de cela: ils ont l'instinct de ce qui convient à leurs intérêts et de la façon de contourner les obstacles. 
Ce que nous disséquons ici, ce ne sont pas nécessairement des conceptions qui ont été appliquées; c'est tout simplement le sens, le chiffre des choses, tels que les circonstances diverses de la conquête de 1760 , les événements complexes qui l'ont suivie, et la situation des colonies de l'Amérique du Nord britannique qui en est résultée en 1867, qui en ont disposé le déroulement. Mais un meilleur plan ne pouvait être conçu pour éliminer graduellement les résistances encore vivaces en 1867 des paysans occupant les terres conquises de 1760 . Car selon ce plan ne pouvaient que se continuer, dans cette population prolifique, l'incapacité de donner du travail à tous les fils du pays sur le sol "national" et par suite l'exode déjà amorcé vers les États-Unis. Et ici le dessein humain s'affirme: on ne fera rien pour favoriser à ces gens le transfert vers cette région cle l'Ouest promise à un avenir prospère. Il faudra que l'Église s'en mêle par pitié pour la misère de toute une population et par le souci de s'en assurer la fidélité, que les paysans pauvres se soutiennent par leurs propres quêtes paroissiales pour payer le gros prix aux chemins de fer afin de suivre le conseil du "Go West young man!" pendant que les citoyens de Varsovie pourront atteindre Winnipeg pour la somme de $\$ 25$. Le capital britannique, qui paraît-il ne mêle jamais le sentiment aux affaires, n'estimait pas que l'argent d'un paysan de la vallée du Saint-Laurent fût de l'odeur qui convenait dans la caisse des chemins de fer assoiffés de revenus, même au prix plus élevé qu'il en coûtait pour obtenir l'établissement d'un paysan de Pologne.

Ce sont les circonstances de l'intérêt américain pour les ressources naturelles du Québec qui déjouèrent en quelque sorte ce plan inconscient dans lequel rien n'était prévu de pareil pour le Québec. Ce fut la revanche des relations nord-sud, plus naturelles que les relations est-ouest qu'essayait de réaliser l'Empire pour assurer la continuité de son prestige en Amérique du Nord. Et peut-être faut-il finalement, à la réflexion, se montrer plus indulgent - quoique pas trop, simplement comprendre sans excuser - pour cette décision prise à Québec, à la fin du XIXe siècle, et qui devait être la règle fixe de 40 ans de régime du parti libéral sous Marchand-Parent, Gouin et Taschereau succes.- 
sivement, de donner littéralement la "Province" aux Américains pour qu'ils y créent des sources de travail au moins au niveau des besoins les plus élémentaires afin de retenir sur place une population attachée à son sol et à ses traditions. Devant la situation tragique où se trouvait le peuple canadien-français, la saignée qui s'y poursuivait systématiquement, peut-être n'est-il pas si facile de garder la tête plus froide que nous avons aujourd'hui sur ces sujets, d'oser poser des conditions un tant soit peu raisonnables à la concession des ressources pour exploitation privée. Il faut penser que toute négociation, avec ses aléas et ses retards, signifiait du temps qui passe et pendant lequel des centaines de Canadiens français traversaient la frontière, annulant tout l'effet de notre seule chance de survie: la "revanche des berceaux".

Car nous touchons là le point sensible de toute cette évolution économique: il n'y avait pas en jeu dans ces décisions, dans ces plans implicites qui en résultaient, dans ces politiques conscientes et contradictoires, seulement le problème banal de l'aménagement d'un espace géographique d'une façon ou d'une autre. Il y avait l'affrontement de deux peuples, l'un conquis, l'autre conquérant; l'un dominé, l'autre dominateur. Sans cela, le goût de développer plus ou moins vite le Québec, plutôt que le reste du Canada, fût resté un pur problème d'intérêt politicoéconomique, ou presque si l'on admet tout de même que toute régionalisation comporte sa part de patriotisme digne de respect dans certaines limites. Selon l'optique des coûts et des rendements économiques, le modèle de développement qu'a connu le Canada est du type le plus onéreux, le moins rentable à court terme; mais il se trouvait parfaitement justifié en fonction d'une considération politique aussi importante que la nécessité d'une protection militaire. Dans un pays à population homogène, le choix ainsi fait serait en somme sans portée majeure en dehors des contraintes économiques qu'il pose au développement. Mais tel n'était plus le cas à partir du moment où le Québec est un territoire "national", dont le développement propre est nécessaire pour permettre l'existence et l'épanouissement d'un peuple en expansion, alors que le reste du Canada constitue le territoire "national" de l'autre peuple. Les phases du développement 
corrélatif des deux territcires ainsi distingués conditionnent tout l'équilibre des relations entre ces deux peuples.

Puisque l'analyse de la question économique sous la Confédération ne peut pas être envisagée du seul point de vue des jeux d'espaces non politiquement qualifiés, comme si le pays eut été composé d'une population homogène, considérons-la maintenant, à partir de cette base où se sont trouvés opposés le Canada et le Québec, en y introduisant la variable "nationale" du groupe français.

Sous ce jour, le plan implicite selon lequel la Confédération engageait le développement canadien était défavorable au groupe national français, en ce qu'il impliquait sa dispersion. En effet, l'évolution normale du plan, avec l'insuffisance de développement économique qu'il postulait pour le Québec, mettait en jeu la mobilité de la main-d'œuvre et la répartition des populations excédentaires du Québec tout le long de la fameuse ligne de chemin de fer, dans l'Ouest, avec un maximum de cristallisation au centre ontarien. Effectivement, il s'est produit une partie de ces déplacements, d'où l'apparition des problèmes des "minorités françaises" au Canada. Mais nous avons vu que la politique du gouvernement central a freiné ce mouvement, dans son désir de contenir le fait français à la "réserve" québécoise, provoquant le phénomène plus grave de l'émigration vers les Etats-Unis.

Toutefois, le problème n'est pas si simple. S'il n'y avait pas eu la Confédération, ou si dans la Confédération le plan de développement du Canada eut suivi ce qu'on peut appeler le mode normal décrit précédemment, tout l'impact du peuplement et du développement se fût centré sur le Québec et l'Ontario. La population francophone au Québec serait peut-être de l'ordre de sept à huit millions ou plus, mais à combien s'élèverait la population totale? Il n'y a pas de raison de supposer que la population totale du Québec et de l'Ontario ne serait pas égale à la population actuelle du Canada; peut-être plus forte même, car ce développe- 
ment plus intensif et moins onéreux aurait pu assurer un bien plus grand développement encore aux deux "provinces". Et alors, notre situation comme groupe national ne se trouverait-elle pas en définitive beaucoup plus mauvaise qu'elle ne l'est à l'heure actuelle?

Si les moments de ce développement avaient été des temps de planification, c'est en somme devant un dilemme que se seraient trouvés les planificateurs de l'avenir du Canada français. La circonstance de la menace militaire qui a dicté le plan implicite du développement économique du Canada nous a donc peut-être fort bien servis. Elle a rendu économiquement naturelle la préservation de cette "réserve" québécoise dont nous avions besoin pour survivre en conservant un foyer "national" de vie politique. Le conquérant a dû aller établir ailleurs le centre et la direction de sa colonisation. Le Québec français s'est trouvé ainsi dégagé d'une pression plus forte, plus impérieuse et plus irrésistible d'intégration et d'assimilation qui aurait autrement été nécessaire au pouvoir dominant pour réaliser ses objectifs de peuplement et de mise en valeur.

Mais le dilemme ne s'arrêtait pas là. Car cette situation comportait en elle-même son germe de mort. Dans le cadre confédératif, dans l'état économique qui était alors celui des Canadiens français, il leur était impossible par eux-mêmes de bâtir assez vite une économie qui aurait pu rivaliser avec le progrès économique du reste du Canada et faire vivre au Québec tout l'excédent de croissance de la population. Bien sûr, il aurait été possible de faire mieux que ce qui a été fait en galvanisant les énergies, en créant une canalisation de l'épargne pour le développement par l'action gouvernementale et la création de banques d'affaires, en organisant le plus possible toute la population selon le mode coopératif. Mais notre pouvoir économique du temps n'aurait pas suffi à créer une économie assez puissante pour rivaliser avec l'économie du groupe anglophone, surtout dans l'opposition qu'elle aurait sans cesse rencontrée des effets contradictoires de la politique canadienne. Sans doute, notre évolution économique y aurait gagné énormément si plus de sens de fierté nationale 
nous eût incités à cela; nous nous serions donné au moins une base de lancement plus valable pour les temps actuels. Mais il nous fallait vraiment pour conserver la force du nombre une mise en valeur intensive qu'exigeait cette invasion d'un capital étranger que nous avons connu et qui apportait avec lui ses propres dilemmes de la dégradation à long terme de la conscience ou du sens national. C'est l'appui de la France qu'il nous aurait fallu comme Mercier l'avait compris.

A la vérité, la Confédération a tout de même contribué pour une part à la conservation du nombre en notre faveur grâce au fait accidentel de la situation de Montréal en terre québécoise. Car Montréal est d'abord un hasard de l'histoire avant que d'être une création humaine agglomérée autour de ce hasard; c'est bien un hasard qui a fait que les rapides Lachine se trouvent là où ils sont et qu'à cause de cela Montréal était appelée à devenir le port ultime d'entrée et la métropole du plan de développement canadien axé vers le centre et l'ouest. C'est un fait qu'après la Confédération, ill y eut un ralentissement sensible de l'émigration des Canadiens français vers les Etats-Unis. Mais le mouvement avait repris à la fin du XIXe siècle; et c'est dans un esprit de panique que les gouvernants du Québec se disposèrent à aider au développement économique par l'accueil au capital américain, dont les excédents déferlaient à nos frontières dans le souci d'approvisionner l'industrie américaine de matières premières.

Au moment de la Confédération, il y a des indications que les Canadiens français avaient relevé la tête dans l'ordre économique et qu'ils n'étaient plus le peuple exclusivement rural ou presque qui avait émergé de la Conquête. Etienne Parent, dans son "Discours devant l'Institut canadien" en 1850, signale que depuis 1840 la "classe des marchands" a connu "un essor tout à fait encourageant pour les autres branches de l'industrie". Ils ont cessé d'être "les agents secondaires des marchands bretons" et ils traitent maintenant directement avec les manufacturiers 
et les marchands des lles britanniques. Il mentionne aussi nos "industriels aisés".

"L'essor tout à fait encourageant" que les progrès du commerce faisaient prévoir à Parent semble bien s'être produit après la Confédération, dans les années 1875 à 1910. L'Histoire de la Province de Québec de Robert Rumilly a recueilli les noms des hommes d'affaires canadiens-français qui prennent leur bonne part de l'activité économique du Québec. Louis-Adélard Sénécal, considéré comme le pair de sir Hugh Allan, l'homme du Pacifique Canadien, Guillaume Boivin dans la chaussure, Joseph Barselou dans les savons, F.-L. Béique à l'origine de notre industrie hydroélectrique, Alfred Dubuc, rival de Price dans les pâtes et papier, Joël Leduc, dans le sciage du bois, Édouard-Alfred Lacroix, fondateur de la North Shore Power, J.-N. Rolland, dans le papier, Louis-Joseph Forget, animateur des "trusts" de l'électricité, des textiles, de l'acier, du ciment, etc.

Mais le gouvernement n'aura pas assez entendu les recommandations d'un Errol Bouchette sur la nécessité d'assurer et de protéger la propriété industrielle autochtone en étayant par une "organisation puissante, appuyée d'une politique industrielle de la part des pouvoirs publics". Aussi non seulement l'entrée du capital américain permettra-t-il un développement plus rapide de la mise en valeur des ressources naturelles, mais il signifiera une rafle générale des entreprises. Et de 1910 à 1914, les noms canadiens-français qui apparaissent au conseil d'administration des grandes affaires ne sont plus ceux des hommes d'affaires, mais des ministres du cabinet provincial ainsi récompensés pour leur générosité envers le capital étranger.

Pendant cette période, les gouvernants avaient aussi commencé à entendre les propos plus anciens de Parent, repris par Errol Bouchette, sur la nécessité d'un haut enseignement technique et commercial. Et c'est alors qu'après l'École polytechnique déjà vieille de plus d'un quart de siècle, naissent les écoles techniques et l'École des Hautes Etudes commerciales. Mais l'invasion du capital américain et la latitude qu'on lui laisse d'occuper toute la place vont singulièrement neutraliser l'influence de 
ces mesures salutaires. Les nouveaux occupants n'ont effectivement guère cure d'utiliser les hauts techniciens du monde francophone: ils "importent" les leurs; et les diplômés de nos grandes écoles deviennent plus ou moins des gens frustrés de se voir fermées inexorablement les avenues où leur talent et leur formation leur permettraient d'aller. Et cela n'est pas seulement un malheur personnel mais bien national, car le savoir inutilisé reste un savoir livresque qui n'engendre aucune tradition de travail à transmettre aux générations suivantes pour créer progressivement ce "know-how" indispensable au progrès d'une collectivité. C'est donc péniblement que les Canadiens français réussiront à s'infiltrer dans les grandes affaires, comme goutte à goutte, par le biais d'une de ses dimensions professionnelles: la comptabilité publique.

A la même période encore naissaient au Canada français les premières expériences de coopératisme, si particulièrement bien adaptées à la situation du peuple canadien-français. C'est en 1900 qu'Alphonse Desjardins organisait, à Lévis, la première caisse populaire; et les coopératives agricoles voyaient le jour quelques années plus tard. Malheureusement, le secteur de la consommation ne connut pas, à ce moment-là, le démarrage qui nous eût peut-être été salvateur. Et quand il s'amorça, beaucoup plus tard, dans les années '30, sous l'impulsion de Victor Barbeau et de Madame Louard, la grande prospérité, si relative qu'elle fut, celle que nous avait amenée l'initiative américaine en nous élevant à proximité de son propre standard de vie, avait déjà trop gâté notre population pour qu'on la trouvât vraiment consciente de sa "misère" profonde, avant tout patriotique, psychologique, et prise du désir intense de prendre en mains son propre destin. La preuve se trouve cependant faite par l'histoire que le mouvement coopératif a été la seule initiative qui nous a permis de déboucher sur des réalisations importantes et stables dans le domaine économique. Car, à bien dire, le mouvement coopératif de crédit avec son milliard et demi et les coopératives agricoles avec leur chiffre d'affaires de plus de 200 millions sont tout ce que nous avons de vraiment grand, qui n'ait jamais bronché et qui montre des progrès constants. Je sais que d'aucuns penseront 
ici à l'Hydro-Québec, mais cette entreprise étatisée n'est que le rachat par l'Etat d'une entreprise montée de toutes pièces par l'initiative et le capital étranger.

Quand Victor Barbeau, en 1936, fit le bilan de notre situation, dans sa Mesure de notre taille, il n'eut pas à faire grand état de la place du mouvement coopératif dans la description de notre situation économique, car les progrès à cette époque n'étaient pas encore de nature à épater. Il n'eut pas de difficulté à marquer notre absence à peu près totale de tout ce qui comptait alors dans le secteur capitaliste, mesurant ainsi une régression réelle par rapport à l'avant-guerre 1914: un monde économique nouveau s'était construit au Québec sans nous et en nous enlevant une bonne partie de ce que nous avions déjà; de sorte que ce qui relevait en propre de l'économie canadienne-française ne donnait que la figure d'initiatives marginales, flottantes, fluctuantes, sans autre portée ou signification que les avantages personnels qui en pouvaient temporairement résulter pour quelques-uns. Il y a eu quelque progrès par rapport à ce temps, mais nos "empires" montrent encore des signes de faiblesses et d'instabilité qui ne permettent pas d'être trop affirmatifs quant à leur pérennité. Et de l'ouvrage que vient de publier Rosaire Morin sur nos Réalités et perspectives économiques, il nous faut tirer que si nous avons paru, dans le secteur financier, accroître dernièrement notre importance dans l'ensemble, nos progrès en chiffres absolus des dernières années cachent un recul constant dans l'ordre proportionnel.

En 1931, les statistiques du recensement avaient montré que sous le seul aspect du nombre, sans tenir compte de l'importance respective des entreprises, dont nous savons déjà qu'elle jouerait en notre défaveur, nous n'occupions une place proportionnelle à notre population dans la direction de l'économie que dans l'agriculture, l'abattage du bois et les services. Dans le commerce et les transports, nous comptions pour les $2 / 3$ du nombre alors que nous étions les $3 / 4$ de la population; et nos proportions décroissaient rapidement avec l'importance significative des secteurs: $56 \%$ dans le secteur manufacturier (avec des proportions dé- 
croissantes selon l'importance des catégories manufacturières), $53 \%$ dans les mines, $48 \%$ dans la finance. Avec des carences comparables dans le secteur professionnel (76\% dans les professions libérales, $41 \%$ dans les professions industrielles et commerciales) et chez les ouvriers techniciens ${ }^{1}$.

Quand M. Allen a repris cette étude, avec les chiffres des recensements de 1951, il n'a pas trouvé en progrès les Canadiens français du Québec selon cette optique. Bien sûr, toujours la même prédominance numérique dans l'agriculture et dans l'abattage du bois; pas de changement dans le commerce et les transports; dans les manufactures, une perte sensible de $8 \%$ à 44 ; dans les mines, perte sensible aussi de $9 \%$ à 44 ; dans la finance cependant un gain assez net de $13 \%$. Toutefois les pertes sont accentuées, et les gains ou les maintiens atténués par le fait que nous n'étions plus alors les $3 / 4$, mais les $4 / 5$ de la population. ${ }^{2}$

Curieuse est donc l'histoire économique de ce pays qu'a été le Canada sous le régime confédératif. Une histoire dont personne, en définitive, n'est satisfait, sauf l'Ontario, qui se plaint cependant aujourd'hui d'être indûment taxée pour assurer aux autres provinces une péréquation de revenu qui leur permette d'aspirer à l'égalité de services. Cette histoire, en définitive, ne fait que ratifier les thèses de Siegfried. Le Canada comme tel n'a été fondamentalement qu'un dessein politique impérial, conçu en fonction des intérêts de l'Empire, à l'encontre des données de la géographie et de la polarisation des espaces économiques. Et l'on peut sérieusement se demander si sans la menace américaine qui justifiait le dessein politique, la "prospérité des provinces" ne se serait pas trouvée beaucoup mieux assurée si les colonies de l'Amérique du Nord britannique étaient restées identifiées, ou en tout cas unies en trois ou quatre grands blocs régionaux qui auraient $\mathrm{pu}$ chercher leur destin propre dans les relations

${ }^{1}$ L'Actualité économique (octobre 1939).

2 Patrick Allen, dans L'Actıalité économique (avril-juin et juilletseptembre 1962). 
naturelles avec l'extérieur, dont principalement les Etats-Unis. $\mathrm{Au}$ fond, les conditions naturelles ne pouvaient pas être plus mauvaises pour tenter, dans l'espace canadien, une expérience d'intégration économique selon les lois de l'économie classique.

Tant que le sentiment impérial a été fort au Canada même - et ce fut le cas jusqu'à tout récemment - c'est en fonction des avantages impériaux que chaque Canadien anglais considérait l'expérience canadienne. Ces inconvénients se payaient alors d'un certain prix qui était facilement accepté comme une contribution valable à la grandeur impériale. Mais à partir du moment où ce sentiment s'est effacé, on comprend que le Canada anglais se soit mis à s'interroger sur l'opportunité même du Canada, cette patrie qui n'en avait jamais été une pour lui que par rapport à un ensemble plus vaste dont le centre était situé ailleurs et qui, considérée en elle-même, paraît coûter extrêmement cher en termes de possibilités économiques et de standard de vie. Il est ainsi normal que 100 ans après 1867, on trouve le Canada anglais comme divisé en deux camps: ceux qui ayant développé une fierté proprement canadienne s'insurgent maintenant contre la domination américaine et ceux qui, prêts à se faire des ÉtatsUnis une patrie autant qu'autrefois l'Angleterre, favorisent les solutions économiques d'intégration, sans trop se préoccuper si au bout il n'y aura pas finalement l'intégration politique.

Mais les Canadiens français du Québec n'en sont pas là, même si c'est encore trop leur instinct bien plus que le sens d'une véritable fierté nationale qui les fait tenir à ce qu'ils sont, parfois contre toutes les apparences que manifeste leur comportement souvent irrationnel. Eux, ils cherchent des explications à la vanité de leurs efforts, à ces perpétuels recommencements comme à zéro; et beaucoup d'entre eux sont tout simplement défaitistes devant tout ce qui prétend s'affirmer pour s'extirper des grands courants fatalistes. La Confédération est, parmi bien d'autres, l'un des facteurs que les uns tendent à incriminer pour tout, alors que les autres, de moins en moins nombreux, se rangent aux thèses officielles et $\mathrm{y}$ voient la planche de salut. 
Nous avons vu qu'il y a de quoi entretenir la confusion, car la Confédération constitue, pour le Canada français du Québec, un paradoxe et pose quelques dilemmes. Et ces dilemmes tendent à indiquer que la clef du problème n'est pas là, mais bel et bien dans la Conquête et dans ses conséquences. Pour pouvoir assurer son expansion économique sans risque d'écrasement pour le $\mathrm{Ca}$ nada français, il aurait vraiment fallu que le Québec fût libre. Libre de diriger son peuplement afin de proportionner les flots d'immigrants à ses propres exigences de développement et d'assimilation; comme de proportionner son développement aux exigences de son expansion démographique; à défaut de cette liberté, mieux lui a probablement valu la Confédération, avec son ouverture et ses appels vers l'Ouest pour le peuplement britannique, que d'être colonie indépendante dont les dirigeants impériaux auraient quasi forcément voulu faire une colonie de peuplement et auraient poussé le développement en fonction des intérêts britanniques. Libre aussi d'avoir sa politique nationale en vue de conditionner le développement selon les intérêts spécifiques de la population du territoire québécois, en fonction de quoi la Confédération a constitué une entrave majeure mais sans qu'on puisse voir s'il y avait vraiment une autre issue qui, parmi les choses possibles, pouvait être plus satisfaisante.

FrançoIs-Albert ANGERS 